\title{
Alerting Threshold Analysis of TCAS
}

\author{
Hongya Tuo ${ }^{1,2, *}$ and Haomiao Zhang ${ }^{2}$

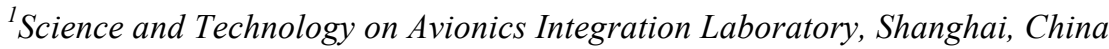 \\ ${ }^{2}$ School of Aeronautics and Astronautics, Shanghai Jiao Tong University, Shanghai, China
}

\begin{abstract}
A method for the setting and evaluation of TCAS (Traffic Alert and Collision Avoidance System) alerting threshold is proposed in this paper. Firstly, by analyzing the error and uncertainty of the flight path, a stochastic differential equation is introduced to model the random aircraft motion. Then a pilot model according to ICAO standards is given to simulate the TCAS alert event. And then five kinds of alarm outputs are summed up by analyzing the principle of TCAS alerting function. Finally, the evaluation system with multiple variables is established on System Operating Characteristic (SOC) curve. The validation of the Encounter model is performed under different parameters. Through the simulation under different threshold values, the optimal alerting threshold values are obtained. The experiments show the rationality of the TCAS threshold and verify the validity of our threshold-setting method.
\end{abstract}

Keywords: TCAS (Traffic Alert and Collision Avoidance System), Alerting threshold, System Operating Characteristic (SOC) curve.

\section{INTRODUCTION}

Currently, air traffic collision avoidance system mainly includes Air Traffic Control system (ATC) based on the ground radar equipments and autonomous airborne Traffic Alert and Collision Avoidance System (TCAS). Different from ATC for the long distance conflict detection, TCAS focuses on the short-term conflict detection. TCAS can provide Traffic Advisory (TA) and Result Advisory (RA) depending on the circumstances surrounding the aircraft in order to avoid the collision of aircrafts and ensure the aviation safety. TA gives the traffic situation to the pilots for reference and does not force them to take some actions. While RA gives "Climb" or "Descend" instructions, and force the pilots to take evasive actions according to the instructions.

The existing conflict detection of TCAS is based on the deterministic model which uses the remaining safe time $\tau$ as the alarming measure. $\tau$ is defined as estimated time when two airplanes meet at the closest point of approach( CPA), which unit is second. The horizontal $\tau$ equals to the horizontal distance between the two airplanes divided by the horizontal approaching velocity. The vertical $\tau$ is with respect to vertical distance and velocity. Only when both the horizontal and vertical approaching times are reached at the threshold $\tau$, TCAS will give the corresponding TA or RA alarm [1]. Herein we discuss the RA warning alarm.

\footnotetext{
*Address correspondence to this author at Science and Technology on Avionics Integration Laboratory and Shanghai Jiao Tong University, Shanghai, 200240, P.R. China; Tel: 13918090553; E-mail: tuohongya@gmail.com
}

Many researchers have studied on the conflict detection algorithms of TCAS. Kuchar and Lee Y [2, 3] made much statistical analysis on a large number of simulation data and gave the safety assessment of the TCAS versions (TCAS6.04a, TCAS7.0, and TCAS7.1). Hu Jianghai, Maria Prandini and John Lygeros $[4,5]$ focused on the long distance conflict detection algorithms of ATC, including the flight path modelling based on Markov chain and hazard detection used on Brownian motion. In recent years, Kochenderfer conducted the extensive research on the TCAS. They built the encounter model of TCAS based on Bayesian network [6], and analyzed the collision probability [7]. They introduced the uncertainty of the flight into the collision model [8] which effectively improved the flight safety. Kwok-On Tong made the in-depth study [9] on the parallel approaching of TCAS. Selim Temizery [10], Evan Maki [11], Hyunjin Choi [12], Lin, C.E. [13] focused on the modeling and conflict detection for UAV. Cui Deguang [14], Lin Yunsong [15, 16] and Feng Ziliang [17] proposed a variety of conflict detection methods and collision avoidance model based on geometric theory for ATC and TCAS.

The work presented in this paper includes the following contents. Based on the analysis of TCAS alert deterministic algorithm, we consider the uncertainty of the flight path and the pilot's reaction to build the avoidance model. Then we simulate the TCAS alerting events to get the optimal threshold.

\section{MODELING OF TCAS ALERTING EVENTS}

In this section we first analyze the error of the flight path together with the uncertainty of the flight path. Comprehensively considering the uncertainty, we use difference equation and proper parameters of the transfer matrix to build the 


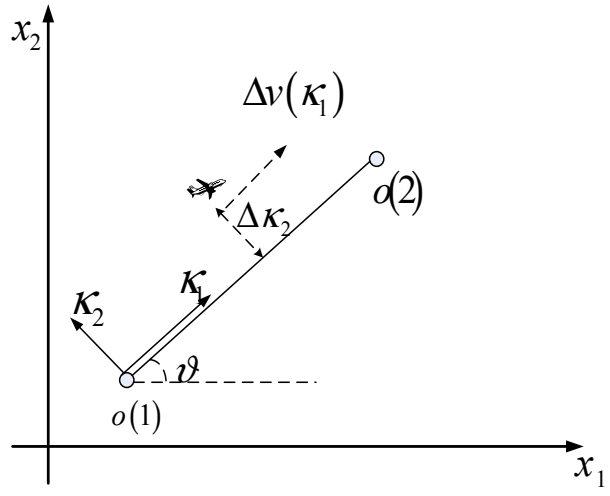

Fig. (1). Coordinate of flight path.

random flight path generating model, which is approximately practical and required by the simulation. Based on the model, we take the reaction delay of the pilot into account and build the after-alert avoidance model based on the standard operation, and then we take advantage of the random path generating model and the avoidance model to simulate the TCAS alerting.

In a TCAS alerting simulation, we first extrapolate the path according to the current state of the aircraft and the random path generating model. If there are conflicts between paths, and the estimated conflict time reaches the safe time $\tau$ of TCAS, the alert is given. After the alert, we simulate the avoidance of the aircraft according to the after-alert avoidance model, and compare the avoidance path with the former. If the avoidance path still has confliction, a collision accident might happen, and vice versa.

\subsection{Analysis of Flight Path' Error}

When an airplane is flying, it is affected by aircraft performance parameters, flight intentions, flight plan and spatial environment. So the flight path is uncertainty. In short-term path forecast, spatial environment is the main factor, including wind, temperature, and cloud. Theoretically, according to the central limit theorem, a variable composed by a large number of small and independent random factors can be considered as a normal distribution. Erzberger [18] made experiments to verify this point through analyzing more than 4000 real airplane's path datum.

Fig. (1) shows the coordinate diagram of the flight path. $O(1), O(2)$ are the two route points. The line through $O(1)$ and $O(2)$ is defined as $\kappa_{1}$ axis, and the vertical line of $\kappa_{1}$ is $\kappa_{2}$ axis. Let $\kappa=\left(\kappa_{1}, \kappa_{2}\right)$ be the position of the airplane under the new coordinate. The actual flight direction is roughly consistent with the $\kappa_{1}$, and vertical to $\kappa_{2}$.

In the two-dimensional coordinate system, we can consider separately the trajectories along $\kappa_{1}$ and $\kappa_{2}$ directions. The flight management system (FMS) controls aircraft flying along a predetermined route by using the velocity deviation $\Delta v\left(\kappa_{1}\right)$ as the feedback at $\kappa_{1}$ axis, and the position deviation $\Delta \kappa_{2}$ as the feedback at $\kappa_{2}$ axis. So the variance $\sigma_{a}^{2}$ of the trajectory error is proportional to the square of time $t$ at $\kappa_{1}$ axis:

$\sigma_{a}^{2}(t) \sim r_{a}^{2} t^{2}$

The variance $\sigma_{c}^{2}$ of the trajectory error is proportional to the square of trajectory $s(t)$ at $\kappa_{2}$ axis. As the feedback at $\kappa_{2}$ axis is position deviation, there exists a limit value $\bar{\sigma}_{c}^{2}$ which controls flight not to have much deviation along $\kappa_{2}$ axis. So we have:

$\sigma_{c}^{2}(t) \sim \min \left\{r_{c}^{2} s^{2}(t), \bar{\sigma}_{c}^{2}\right\}$

\subsection{Random Trajectory Generating Model Based on Gaussian Difference Equation}

Discreting the continuous trajectory with sampling cycle 1 second, we build the difference equation. With proper parameters, the equation can generate the trajectory that approximately satisfies the actual error rules.

Assume under the path coordinate, $\kappa_{1}$ is independent of $\kappa_{2}$, then the position variance $\bar{V}(t)$ in $\kappa_{1}$ and $\kappa_{2}$ coordinate is the diagonal matrix with diagonal elements $\sigma_{a}^{2}(t)$, $\sigma_{c}^{2}(t)$ :

$\bar{V}(t)=\operatorname{diag}\left(\sigma_{a}^{2}(t), \sigma_{c}^{2}(t)\right)$

Let $x=\left(x_{1}, x_{2}\right)$ be the position of the aircraft in the world coordinate, $\vartheta$ is the angle between $x_{1}$ and $\kappa_{1}$, then the transition between the two coordinates is:

$x=R(\vartheta) \kappa+p$

Which $\mathrm{p}$ is the initial position coordinates of the aircraft in the world coordinate. And $R(\vartheta)$ is the rotation matrix,

$R(\vartheta)=\left[\begin{array}{cc}\cos \vartheta & -\sin \vartheta \\ \sin \vartheta & \cos \vartheta\end{array}\right]$

The position variance matrix of the aircraft in the world coordinate is:

$V(t)=R(\vartheta) \bar{V}(t) R(\vartheta)^{T}$

We describe the path of the aircraft using random difference equation:

$\dot{\kappa}(t)=A \kappa(t)+\eta(t)$ 
A is the transfer matrix of the difference matrix, and is time-invariant. $\eta(t)$ represents the random perturbation of the flight path in the flight path coordinate $\eta(t)$, which reveals the uncertainty of the difference equation. Let $\kappa(0)$ be the initial position coordinate of the aircraft in the flight path coordinate. $\eta(t)$ and $\kappa(0)$ are both vectors that follow Gaussian distribution, and the elements are independent $[19$, 20], which is:

$$
\begin{aligned}
& \eta(t) \sim N\left(0, \operatorname{diag}\left(\bar{v}_{\eta 1}(t), \bar{v}_{\eta 2}(t)\right)\right) \\
& \kappa(0) \sim N\left(0, \operatorname{diag}\left(\bar{v}_{\kappa 1}(0), \bar{v}_{\kappa 2}(0)\right)\right)
\end{aligned}
$$

Because the initial position of the aircraft is not revelant to the random perturbation of the flight path, $\eta(t)$ and $\kappa(0)$ are independent.

We take the derivative of (4) and take (7) in:

$$
\left\{\begin{array}{c}
\dot{x}=R(\vartheta) A R(\vartheta)^{T}(x-p)+R(\vartheta)[1,0]^{T} v+R(\vartheta) \eta \\
\dot{p}=R(\vartheta)[1,0]^{T} v
\end{array}\right.
$$

Because the initial position of the aircraft is precise, we let $\bar{v}_{\kappa 1}(0)=\bar{v}_{\kappa 2}(0)=0$ and $\bar{v}_{\eta 1}(t)=2 r_{a}^{2} t$,

$$
\bar{v}_{\eta 2}(t)=2 a \bar{\sigma}_{c}^{2}, a=\frac{r_{c}}{\bar{\sigma}_{c}} v, A=\left[\begin{array}{cc}
0 & 0 \\
0 & -a
\end{array}\right] \text {. We take them }
$$

in (10), then $\kappa_{1}$ and $\kappa_{2}$ are independent and are both zeromean Gaussian stochastic process with variance:

$$
\begin{aligned}
& \operatorname{var}\left[\kappa_{1}(t)\right]=r_{a}^{2} t^{2} \\
& \operatorname{var}\left[\kappa_{2}(t)\right]=\bar{\sigma}_{c}^{2}\left(1-\exp \left(-2 \frac{r_{c}}{\bar{\sigma}_{c}} v t\right)\right)
\end{aligned}
$$

We can see that the random path generating model uses Gaussian difference equation to describe and generate the path, and Gaussian distribution represents the uncertainty of the path.

(11), (12) and (1), (2) are approximately the same, which indicates that the path generated by the model is close to the practical situation.

\subsection{Model of the Avoidance Process}

After the TCAS warning is given out, the avoidance process can be divided into three phases: delay stage, pull-up stage, and steady climbing stage. The first stage is determined by the pilot's delay time $t_{\text {delay }}$. And the uncertainty of $t_{\text {delay }}$ can be described by the random probability models. Through a lot of statistic analysis based on the actual datum, the gamma distribution could be approximately used as the models of $t_{\text {delay }}[2,6]$.
In the pull-up and steady climbing stages, the models should be in accordance with the avoidance actions recommended by ICAO (International Civil Aviation Organization ). At first, the airplane will be pulled up with acceleration of $0.25 \mathrm{~g}$ till the vertical speed attains $1500 \mathrm{ft} / \mathrm{min}$, then keep the vertical speed and enter into the last stage. Only when the vertical distance between two airplane reaches to $600 \mathrm{Ft}$, no alarm of TCAS will be sent out.

During the avoidance process, assume that the two conflicting airplanes take cooperative actions. That means they take avoidance actions in the opposite vertical direction while holding their horizontal speed unchanged. Define $t_{\text {avoid }}$ as the time used in the pull-up and steady climbing stage till no alarm is given out. So to successfully alarm, the safety threshold time $\tau$ should be bigger than the sum of $t_{\text {delay }}$ and $t_{\text {avoid }}$.

\section{EVALUATION METHODS FOR TCAS SYSTEM}

Based on the deterministic analysis about TCAS alert, five alarm outputs can be summarized. System operating characteristic curve can be used to give an evaluation on TCAS warning system

\subsection{TCAS Output}

Consider the three factors, flight path state, an alert whether to be sent out and the effect of the alert, there are five outputs of TCAS system:

(1)Insuccessful Alarm (IA): TCAS sends out a warning in a dangerous situation, but is too late to avoid the conflict.

(2)Successful Alarm (SA): TCAS sends out a warning in a dangerous situation, and succeeds to avoid the conflict.

(3) Missing Alarm (MA): TCAS doesn't send out a warning in a dangerous situation.

(4) Failure Alarm (FA): TCAS send out a warning in a safe situation.

(5) Correct Depress (CD): TCAS doesn't send out a warning in a safe situation

TCAS system is ideal when in a dangerous situation TCAS sends out a warning and succeeds to avoid the conflict, and when in a safe situation TCAS doesn't send out a warning. But in fact due to the uncertainty of the fight path and the pilot' delay mentioned above, insuccessful and missing alarm still exit in a dangerous situation, and maybe there are failure alarms in a safe situation.

It is critical how to select the safety threshold time $\tau$ for TCAS. If $\tau$ is given too large, this can ensure the safety to some extent, but meantime lead to many failure alarms and influence the pilot greatly. If $\tau$ is given too small, failure alarm will be reduced effectively, but the polit's delay and avoidance time ( $t_{\text {delay }}$ and $\left.t_{\text {avoid }}\right)$ may be close to or greater 


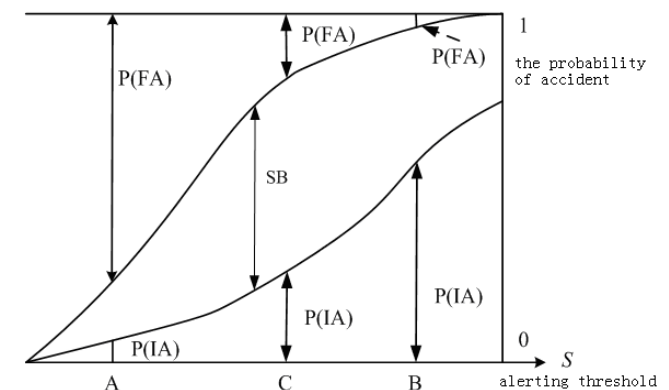

Fig. (2a). PM (Performance Metric plot).

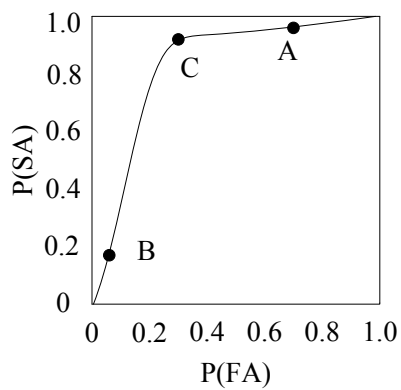

Fig. (2b). SOC(System Operating Characteristic) curve.

than $\tau$, which will increase the insuccessful Alarm. So the selection of $\tau$ needs to balance these two aspects.

\subsection{Evaluation Method Based on SOC(System Operating Characteristic) Curve}

As a typical alarm system, TCAS gives out the alert depending on the current situation. When an alert is sent out, there are three possibilities mentioned above: Insuccessful Alarm(IA), Successful Alarm(SA) and Failure Alarm(FA). Let their probability as $P(I A), P(S A), P(F A)$ irrespectively. So we have

$P(S A)=1-P(I A)$

Define system benefits ( $S B$ ) as:

$S B=1-P(F A)-P(I A)$

As a reasonable alert system, it should be minimized $P(F A)$ and $P(I A)$. But it is difficult to meet all the goals to get the optimal $S B$. We can use SOC method to obtain the threshold and ensure $S B$ in the acceptable range.

Fig. (2) shows the SOC method. Fig. (2a) calls as $P M$ (Performance Metric) curve. The $\mathrm{x}$-axis stands for the alerting threshold, and the y-axis is the probability of accident. The upper is the accident probability curve without alerts, and the lower is the accident probability curve with alerts. Assume A, B and C are different alerting thresholds shown in Fig. (2a). A point corresponds to an earlier alert threshold, so $P(S A)$ is greater but $P(F A)$ is greater too. While $\mathrm{B}$ point corresponds to a later alert one, $P(S A)$ is smaller and

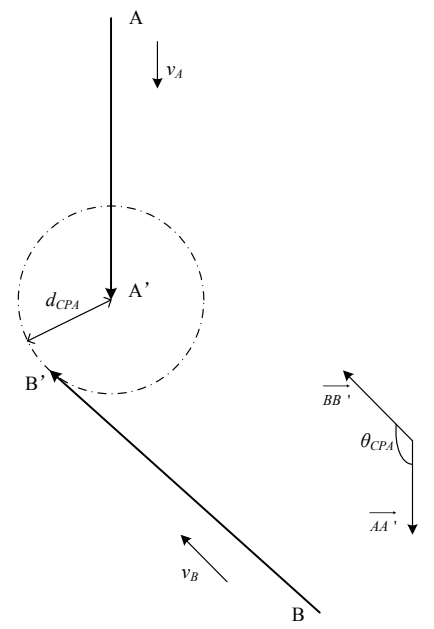

Fig. (3). Encounter model.

$P(F A)$ is also smaller. So the system benefits $(S B)$ of A and $\mathrm{B}$ are not ideal. We see $\mathrm{C}$ point lies between $\mathrm{A}$ and $\mathrm{B}$, $P(S A)$ is relatively larger and meanwhile $P(F A)$ is lower which can maximize the system benefits $(S B)$.

With $P(F A)$ as the $\mathrm{x}$ - axis, $P(S A)$ as $\mathrm{y}$-axis, we can get the SOC(system operational performance) curve as shown in Fig. (2b). Any point on the SOC curve corresponds to an alarm threshold. So we can obtain satisfactory thresholds under different needs [2].

\section{EXPERIMENTS}

We conduct the Monte Carlo simulation according the random trajectory generating model based on the Gaussian difference equation, and get the accident rate curves under different initial conditions. The results are consistent with the expected solution, and verify the validity of the model. Based on the model, we set different alerting thresholds, and use Monte Carlo simulations of TCAS alerting by 10000 times according to the pilot reaction model. Finally we get the accident rate and the false alart rate of TCAS under different thresholds, and evaluate the values using SOC method

\subsection{Experiments for Verifying the Trajectory Model}

In a collision accident, the two aircraft are in the same altitude level, and the dangerous zone can be defined as a circle with center of the aircraft itself and radius $\rho$ of accident threshold [16]. When the distance between the two aircraft is smaller than $\rho$, we regard the situation as occurrence of NMAC.

Suppose A is the native aircraft equipped with TCAS and $\mathrm{B}$ is the intruding aircraft. The collision initial condition includes the time $t_{C P A}$ to reach the closest point CPA, the distance $d_{C P A}$ when reaching CPA, the velocity of two aircraft $v_{A}$ and $v_{B}$, the height of two aircraft $h_{A}$ and $h_{B}$. This paper considers the general case that two aircraft meet at the same height level ( $\left.h_{A}=h_{B}\right)$, as shown in Fig. (3). 


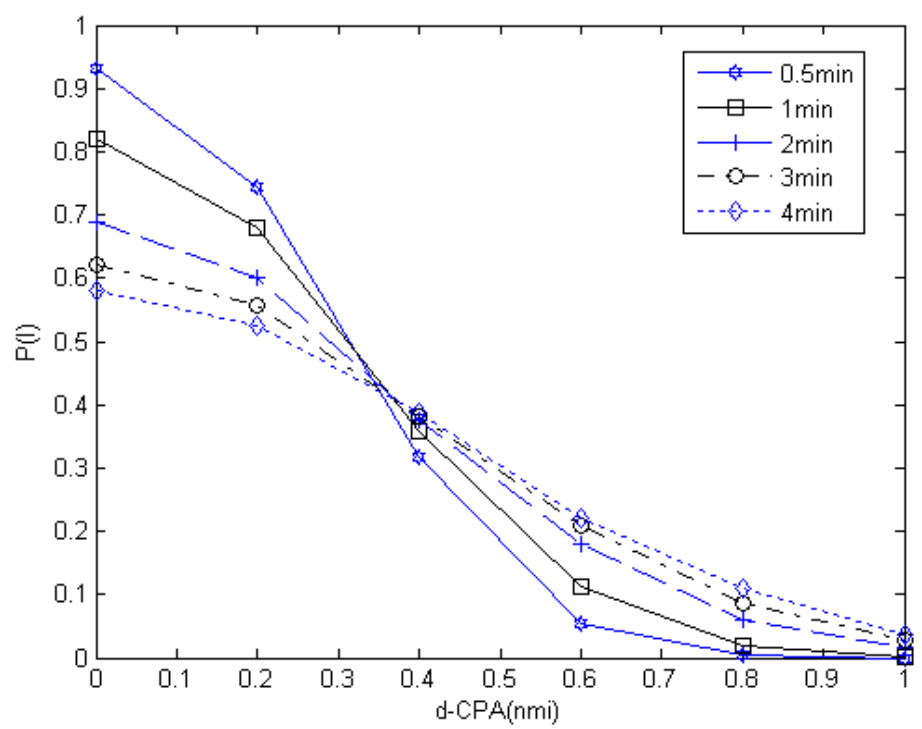

Fig. (4). $P(I A)$ curves under different $t_{C P A}$ and $d_{C P A}$.

We set different initials of the collision model, conduct 10000 Monte Carlo simulations and get the accident rate curve $P(I A)$. We refer to the analysis of the civil aviation trajectory by NASA Ames Research Center, and set the parameters of the random trajectory generating model $\rho$ $=0.3 \mathrm{nmi}, \quad v_{1}=480 \mathrm{kot}, v_{2}=500 \mathrm{kot}$. The accident rate curves under different $t_{C P A}$ and $d_{C P A}$ are shown in Fig. (4).

The results show that the $0.3 \mathrm{nmi}$ is a turning point, and when $d_{C P A}<0.3 \mathrm{nmi}$, with the increase in $t_{C P A}$, the accident rate $P(I A)$ gets lower. Whereas when $d_{C P A}>0.3 \mathrm{nmi}$, with the increase in tCPA, accidents rate $P(I A)$ increases. In the simulation $\rho$ equals $0.3 \mathrm{nmi}$, and with the increase in $t_{C P A}$, the uncertainty of the trajectory that reaches CPA becomes larger. If the originally set $d_{C P A}<\rho$ such that the aircraft reaches CPA as planned, the accident will occur, and in such case when the uncertainty becomes larger, the accident rate will be lower. Whereas if the originally set $d_{C P A}>\rho$ such that the aircraft reaches CPA as planned, then the accident will not occur, and therefore the uncertainty becomes larger, the accident rate will be lower. If we examine every single curve with the same $t_{C P A}$, when $d_{C P A}$ increases, the accident rate get smaller, which shows that when the uncertainty of the flight trajectory remains the same, the larger the distance from the CPA, the lower the accident rate.

The simulation results are consistent with the practical situation, and we further verify the validity of the model generated by the random trajectory based on the Gaussian difference equation.

\subsection{Analysis of TCAS Alerting Threshold Simulation}

The simulation is made on the basis of the collision model, the pilot reaction model, and the standard avoidance operation. The parameters of the random trajectory generating model are $r_{a}=1 / 57, \bar{\sigma}_{c}=1 n m i$. Considering the actual situation of the civil aviation, we determine the parameters of the collision model according to the typical collision accident. Let the arrival time for the two aircraft to reach the desired closest point $t_{C P A}=5 \mathrm{~min}$, the distance $d_{C P A}=0 \mathrm{nmi}$, the angle $\theta_{C P A}=145 \mathrm{o}$, the flight velocity $v_{A}=480 \mathrm{kot}$, $v_{B}=500 \mathrm{kot}, h_{A}=h_{B}=40000 \mathrm{ft}, \rho=0.1 \mathrm{nmi}$ and the alert threshold ${ }^{\tau}$ ranging from $5 \mathrm{~s}$ to $280 \mathrm{~s}$. We make the simulation for every possible $\tau$ with Monte Carlo by 10000 times, and get the statistics curves such as $P(I A)$ and $P(F A)$ with different thresholds. The PM curve is shown as below.

Fig. (6) is the SOC performance curve with different TCAS thresholds based on Fig. (5).

From the PM curve, we can see that with the increase in the alarm threshold $\tau, P(I A)$ becomes small. When $\tau$ reaches $35 \mathrm{~s}, P(I A)=0$, then remains zero. $P(F A)$ decreases too as the alarm threshold $\tau$ increases. When $\tau$ is greater than or equal to $35 \mathrm{~s}$, TCAS can ensure the safety of flight, and with the increase in $\tau$, the false alarm rate P (FA) drops down slowly. When the alarm threshold $\tau$ equals to $35 \mathrm{~s}$, the system gained $S B$ reaches the maximum.

Because of the extreme importance of the safety in the aviation industry, the reasonable threshold of TCAS should optimize $P(S A)$, and based on this, make $P(F A)$ smaller 


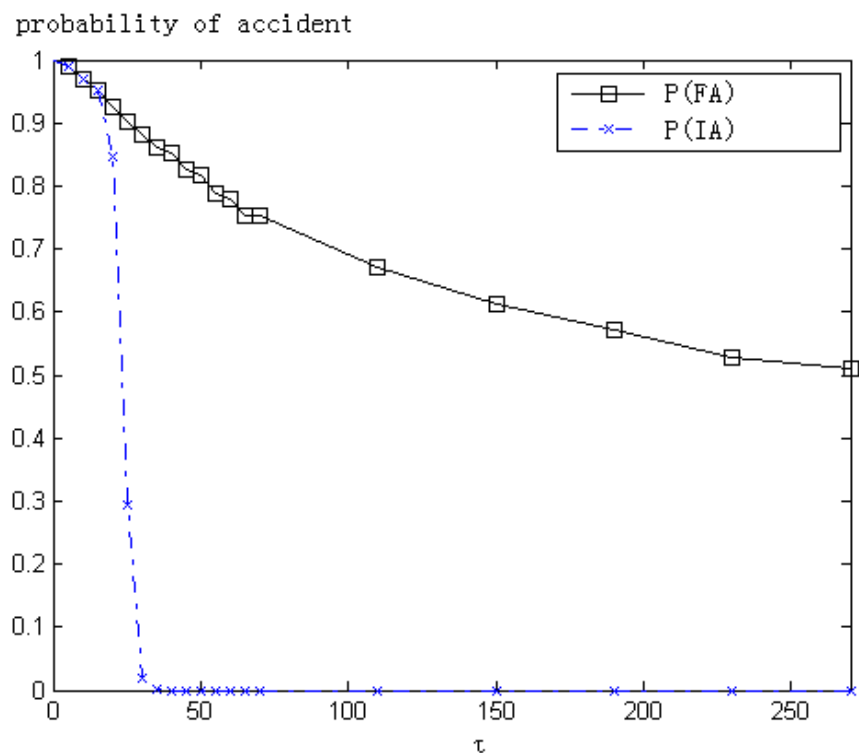

Fig. (5). PM curves under different TCAS alerting threshold value.

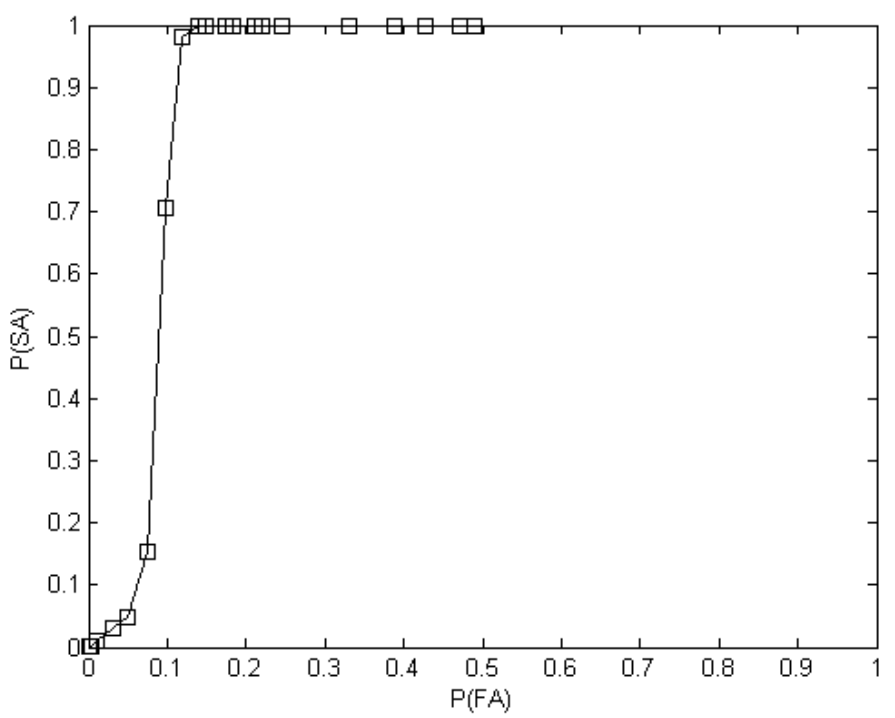

Fig. (6). SOC curve under different TCAS alerting threshold value.

and $S B$ bigger. Therefore the optimal alarm threshold in the simulation environment is $35 \mathrm{~s}$, which is consistent with the threshold in use.

\section{CONCLUSION}

Setting threshold is one of the crucial issues in TCAS researches. A reasonable threshold can optimize the performance alarm warning system. In this paper, we compare the alarm performance with different alarms alarm thresholds by Monte Carlo simulation and SOC method. The optimal alarm threshold in the simulation environment is calculated, and the value is consistent with the threshold in use. The paper evaluates the rationality of the TCAS threshold, and meantime verifies the validity of the threshold-setting mode, so the analysis can be generalized to analyzing the alerting performance of all kinds of TCAS systems.

\section{CONFLICT OF INTEREST}

The authors confirm that this article content has no conflict of interest.

\section{ACKNOWLEDGEMENTS}

This work is supported by the Aeronautical Science Foundation of China (No.20115557007). Meantime the first author is grateful for the financial support from China Scholarship Council (CSC). 


\section{REFERENCES}

[1] RTCA. Minimum Operational Performance Standards for Traffic Alert and Collision Avoidance systemsii(TCASII)Airborne Equipment, //RTCA/DO-185A,1997.

[2] J. K. Kuchar, "Methodology for alerting-system performance evaluation", Journal of Guidance Control and Dynamics(S0731-5090), vol. 19, no. 2, pp. 438-444, 1996.

[3] L.P. Espindle, J.D. Griffith, and J.K. Kuchar, "Safety Analysis of Upgrading to Tcas Version 7.1 Using the 2008 U.S. Correlated Encounter Model", MIT Lincoln Laboratory, Lexington, MA, Project Report ATC-349, 2009.

[4] A. Abate, M. Prandini, J. Lygeros, and S. Sastry, "Probabilistic reachability and safety for controlled discrete time stochastic hybrid systems," Automatica (S 0005-1098), vol. 44, no. 11 pp. 2724-2734, 2008.

[5] J. Hu, M. Prandini, and S. Sastry, "Optimal coordinated maneuvers for three dimensional aircraft conflict resolution," Journal of Guidance Control and Dynamics (S0731-5090), vol. 25, no. 5, pp. 888900, 2002.

[6] M. J. Kochenderfer, L. P. Espindle, James K. Kuchar, and J. D Griffith, "A Bayesian approach to aircraft encounter modeling," In: AIAA Guidance, Navigation and Control Conference and Exhibit, Hawaii , pp. 18-21, 2008.

[7] J. P. Chryssanthacopoulos, and M. J. Kochenderfer, "Hazard alerting based on probabilistic models," Journal of Guidance Control and Dynamics, vol. 35, no. 2, pp. 442-450, 2012.

[8] J. P. Chryssanthacopoulos, and M. J. Kochenderfer, "Accounting for state uncertainty in collision avoidance," Journal of Guidance Control and Dynamics, vol. 34, no. 4, pp. 951-960, 2011.

[9] K. Tong, M. L. Ulrey, and S. R. Conway, "Applications of collision and tcas alerting models in parallel runway operations," In: 10th AIAA Aviation Technology, Integration, and Operations Conference, Texas, pp. 13-15, 2010.

[10] S. Temizery, and M. J. Kochenderfer, "Collision avoidance for unmanned aircraft using markov decision processes," In: AIAA
Guidance, Navigation, and Control Conference, Toronto, pp. 2-5, 2010.

[11] E. Maki, and C. Parry, Dynamic Protection Zone Alerting and Pilot Maneuver Logic for Ground Based Sense and Avoid of Unmanned Aircraft Systems, Aerospace: California, pp. 19-21, 2012.

[12] H. Choi, and Y. Kim, "Reactive collision avoidance of unmanned aerial vehicles using a single vision sensor," Journal of Guidance Control and Dynamics, vol. 36, no. 4, pp. 1234-1240, 2013.

[13] C.E. Lin, Y.H. Lai, and F.J. Lee, "UAV collision avoidance using sector recognition in cooperative mission to helicopters," In: IEEE Integrated Communications, Navigation and Surveillance Conference (ICNS), Herrdon, VA, pp. 1-9, 2014.

[14] D. Li, and D. Cui, "Air traffic control confilict detection algorithm based on Brownian motion," Journal of Tsinghua University(Sci \& Tech), vol. 48, no. 4, pp. 477-481, 2008.

[15] Y. Lin, L. Peng, and L. Tong, "Mathematics model for collision avoidance in traffic alert and collision avoidance system," Journal of University of Electronic Science and Technology of China, vol. 37, no. 4, pp. 552-555, 2008

[16] L. Peng, and Y. Lin, "Modeling and simulation for the model of horizontal collision avoidance in TCAS," Journal of Air Force Engineering University(Natural Science Edition), vol. 11, no. 4, pp. 16-20, 2010.

[17] M. Zha, Z. Feng, and S. Luo, "Mid-term conflict detection algorithm of multi-route conflict probability," Journal of Computer Applications, vol. 30, no. 5, pp.1406-1409, 2010.

[18] A. P. Rusell, and E. Heinz, "Conflict probability estimation for free flight," Journal of Guidance Control and Dynamics (S0731-5090), vol. 20 , no. 3 , pp. $588-596,1997$.

[19] M. Prandini, J. Hu, J. Lygeros, and S. Sastry, "A probabilistic approach to aircraft conflict detection," IEEE Transactions on Intelligent Transport System (S 1524-9050), vol. 1, no. 4, pp. 199-220, 2000 .

[20] A. Pritchett, and S-B. Fleming, "Pilot compliance to TCAS Resolution Advisories," In: Digital Avionics Systems Conference (DASC), NewYork, pp. 6B6-1 - 6B6-13, 2013.

(C) Tuo and Zhang; Licensee Bentham Open.

This is an open access article licensed under the terms of the Creative Commons Attribution Non-Commercial License (http://creativecommons.org/licenses/bync/4.0/) which permits unrestricted, non-commercial use, distribution and reproduction in any medium, provided the work is properly cited. 